\title{
Aspartate aminotransferase in COVID-19: A probably overrated marker
}

To the Editor,

We read with interest the update by Spearman et $\mathrm{al}^{1}$ discussing liver involvement in COVID-19. The review mentioned some results about liver enzymes derived from published studies, discussing the frequently reported association of aspartate aminotransferase (AST) increase with in-hospital mortality risk. This information is potentially relevant for guiding patient care and permitting early identification of subjects at high risk of death, its practical implication being, however, compromised by the fact that no studies discussed how pre-analytical, analytical and post-analytical aspects of laboratory measurements may influence obtained data and their interpretation. Issues such as the lack of standardization of commercial assays towards available international reference measurement systems (eg for aminotransferases, assays with or without the addition of pyridoxal5-phosphate coenzyme are available on the market, providing different results), ${ }^{2}$ the use of upper reference limits (URL) derived from protocols of unknown robustness for defining enzyme increases, ${ }^{3}$ and the unavailability of information about management of results potentially affected by common interferents (eg AST measurements are significantly impacted even when serum samples are slightly haemolysed), all may compromise results obtained in primary studies and make their interpretation ambiguous.

Given the uncertainty of accuracy of published data, we recently examined a large cohort of COVID-19 patients admitted in our academic hospital in Milan, one of the two Italian reference centres for infectious diseases, for defining sources and clinical significance of AST increases, paying attention to all aspects of total examination process. ${ }^{4}$ First, we found AST more frequently increased in COVID-19 patients than in previous studies: $69 \%$ of subjects displayed AST values higher than URL, much more than the pooled prevalence of $19 \%$ reported in Spearman's review. ${ }^{1}$ The pathogenetic mechanisms for abnormal AST frequently present in COVID-19 were likely multifactorial in origin (possibly a combination of hypoxia, hyperinflammation and drug toxicity), having liver and muscles as primary sources in an approximate $60 \%-40 \%$ proportion. More importantly, our data failed to demonstrate an independent association of AST increases with poor outcomes, such as death and intensive care unit admission during hospitalization, as opposed to biomarkers with a strong and widely confirmed prognostic power, such as lactate dehydrogenase and serum albumin. ${ }^{5}$
In conclusion, after having carefully controlled all laboratory sources of variation, we were unable to confirm what other authors have published about the value of AST in COVID-19 as independent prognostic factor. Although quite frequent, AST elevations are probably a disease nonspecific epiphenomenon and have minimal clinical significance.

\section{CONFLICT OF INTEREST}

None.

\section{DATA AVAILABILITY STATEMENT}

Data sharing is not applicable to this article as no new data were generated.

Elena Aloisio

Mauro Panteghini

Clinical Pathology Unit, ASST Fatebenefratelli-Sacco, Research Centre for Metrological Traceability in Laboratory Medicine (CIRME), Università degli Studi di Milano, Milan, Italy Email: elena.aloisio@asst-fbf-sacco.it

Editor: Luca Valenti

\section{ORCID}

Elena Aloisio iD https://orcid.org/0000-0001-6311-5700

\section{REFERENCES}

1. Spearman CW, Aghemo A, Valenti L, Sonderup MW. COVID-19 and the liver: a 2021 update. Liver Int. 2021;41:1988-1998.

2. Infusino I, Frusciante E, Braga F, Panteghini M. Progress and impact of enzyme measurement standardization. Clin Chem Lab Med. 2017;55:334-340.

3. Ceriotti F, Henny J, Queraltó J, et al. Common reference intervals for aspartate aminotransferase (AST), alanine aminotransferase (ALT) and $\gamma$-glutamyl transferase (GGT) in serum: results from an IFCC multicenter study. Clin Chem Lab Med. 2010;48:1593-1601.

4. Aloisio E, Colombo G, Arrigo C, Dolci A, Panteghini M. Sources and clinical significance of aspartate aminotransferase increases in COVID-19. Clin Chim Acta. 2021;522:88-95.

5. Aloisio E, Chibireva M, Serafini L, et al. A comprehensive appraisal of laboratory biochemistry tests as major predictors of COVID-19 severity. Arch Pathol Lab Med. 2020;144:1457-1464. 
How to cite this article: Aloisio E, Panteghini M. Aspartate aminotransferase in COVID-19: A probably overrated marker. Liver Int. 2021;41:2809-2810. doi:10.1111/liv.15068 